(2) When diffusion of ions as well as of non. ionized molecular species takes place across an interphase which is not in a state of equilibrium.

Type 1 maintains a constant charge on its double layer when left on open circuit. For solutions of a given composition, it takes up any potential which is initially imposed. It has no definite potential of its own; but changes in the composition of the solution at constant surface charge produce definite changes of potential which depend on the actual charge on the double layer. These changes of potential arise from the adsorption at the interphase of substances from the solution. The theory of adsorption at interphases of this type has been worked out by Stern ${ }^{2}$, who assumes the validity of Langmuir's adsorption isotherm for such cases. An examination of the partition function for an interphase shows that this assumption is not valid for charged particles ${ }^{3}$, but the general basis of Stern's theory is supported by work on dropping mercury electrodes and electrocapillary data 4 .

Adsorption at interphases of Type 2 will result in general in altered diffusion rates, and may alter these rates differently in the case of different ions. Since the diffusion of neutral electrolytes, interchange of cations and interchange of anions all involve the superposition of electric currents flowing in opposite directions, a selective change in the rates of ionic diffusion may lead to a modified distribution of electric charge. Thus the interfacial potential difference will alter.

The formula for a diffusion potential may be written approximately as :

$$
\Psi_{\beta}-\Psi_{\alpha}=-\sum_{i=1}^{i=8} \int_{\alpha}^{\beta} \frac{t_{i}}{z_{i} F} \cdot d \mu_{i} \ldots . .(1) \text {. }
$$

The integration is from homogeneous solution $\alpha$ to homogeneous solution $\beta$ on the other side of the diffusion boundary, and all the terms have their usual thermodynamic significance. As $\mu_{i}=\mu_{o i}+$ $R T \ln c_{i} f_{i}$ and $\mu_{o i}$ is a constant depending on the temperature, pressure and nature of the solvent, attempts to relate equation 1 to ionic concentrations when water and oil are diffusing into each other involve a consideration not only of the diffusion of each ion but also of the solvent; diffusion of the latter causes the partition coefficients to vary both in space and in time.

As the cells examined by Ehrensvärd and Sillén take up definite potentials, their aqueous - oil interphases cannot be of Type 1. They claim that their observations are consistent with the theory of such an interphase. The early stages of the setting up of a diffusion gradient in oil-water interphases seem therefore to confine the diffusion to the first one or two molecular layers of the oil phase. For these layers, interchange equilibrium is rapidly set up. The subsequent extension of the region of diffusion into the non-aqueous phase is very slow. Kinetically, therefore, a continuous transition can take place from an adsorbed monolayer to a diffusion gradient. Mathematically, Equation 1 is formally valid for a completely polarizable interphase.

$$
\begin{array}{ll}
\text { Cambridge. } & \text { O. GATTY. } \\
\text { May 17. } & \text { ROTHSCHILD. } \\
\text { ' Ehrensvărd, G., and Sillén, L. G., NATURE, 141, } 788 \text { (1938). } \\
\text { ' Stern, O., Z. Elektrochem., 30, 508 (1924). } \\
\text { ' Levine, S., personal communication. } \\
\text { ' Philpot, J. St. I., Phil. Mag., 13, } 775 \text { (1932) ; Craxford, S. R., } \\
\text { Dissertation, Oxford, i936. }
\end{array}
$$$$
\text { S. R. CRaxford }
$$

\section{An Anomalous Case of Emulsification}

IT is commonly supposed that an emulsion of two immiscible liquids is stabilized by the formation of an interfacial film of emulsifying agent molecules which, by their constitution and orientation, condition the emulsion type. Thus, according to the Gibbs' equation, those materials which reduce surface or interfacial tension should facilitate emulsification. Indeed the term surface tension depressor has become synonymous with emulsifying agent.

It has recently come to our notice that simple inorganic salts such as the alkali halides enable relatively stable emulsions of water in amyl alcohol to be produced. These salts are not surface active but tend rather to increase the interfacial tension of the system.

The stability of emulsions thus prepared bears no simple relationship to the concentration of salt in solution. In the case of emulsions stabilized by potassium iodide, the maximum stability appears to be attained at an electrolyte concentration of $0.05 \mathrm{~N}$, while concentrations greater than normal inhibit emulsification completely. On the other hand, concentrations of potassium iodide so low as $0.001 \mathrm{~N}$ have considerable stabilizing action.

D. F. Chemsman.

A. KING.

J. N. Sugden.

Department of Chemistry,

Imperial College of Science and Technology, London, S.W.7. May 20.

\section{Control of Insects by Methallyl Chloride}

IT is difficult to control insects in stored products, where they cause millions of pounds worth of damage annually. The most efficacious method is by fumigating them with poisonous gases or vapours. Many chemical compounds have already been tried, for example, by the United States Department of Agriculture ${ }^{1}$.

I carried out some investigations upon methallyl chloride, and found that this material possesses good insecticidal qualities. It is a product manufactured from petroleum hydrocarbons and has the following formula :

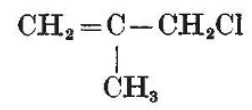

Its specific gravity at $20^{\circ} \mathrm{C}$. is 0.925 and its boiling point $72^{\circ} \mathrm{C}$. It is a colourless liquid which evaporates easily at normal temperature. Mixtures of its vapour with air are not explosive in concentrations of 105-339 gm. per cub. m. It was H. F. Zimmermann at Prague who directed my attention to this product. It was found in the laboratory that species of Ephestia such as $E$. kuhniella Zeller and $E$. elutella Hb. were killed by a concentration of $12 \cdot 5 \mathrm{gm}$. per cub. $\mathrm{m}$. applied for twenty-four hours.

Calandra granaria L., the corn weevil, was killed by a concentration of $18.75 \mathrm{gm}$. per cub. m. applied for twenty-four hours at $18^{\circ} \mathrm{C}$. The insects are not dead immediately after fumigation, but methallyl chloride proved to have a strong after-effect, so that within a few days all the insects are killed. Eggs, larvæ and pupæ are also killed. Imagines of Calandra, placed in the centre of a bag of wheat, were easily reached and killed by the gas. 